\title{
Dedifferentiated Chondrosarcoma with Epithelial Differentiation: A Case Report and Review of the Literature
}

\author{
Motaz AlAqeel, Marc-Antoine Ricard, Humaid Al Farii", Sungmi Jung and Robert Turcotte \\ Department of Orthopaedic Oncology, Division of Orthopaedic Surgery, McGill University, Montreal, Canada
}

\begin{tabular}{l} 
A R T I C L E I N F O \\
\hline Article history: \\
Received: 7 April, 2020 \\
Accepted: 21 April, 2020 \\
Published: 27 April, 2020 \\
\hline Keywords: \\
Dedifferentiated chondrosarcoma \\
epithelial dedifferentiation
\end{tabular}

\begin{abstract}
A B S T R A C T
Case: We describe a case of 72-year-old male with dedifferentiated chondrosarcoma with osteosarcomatous and epithelioid (squamous) dedifferentiation. As first described in the literature by Dahlin and Beabout, a well-differentiated benign chondral lesion abruptly juxtaposed to a high-grade non-cartilaginous component is the classical definition of a chondrosarcoma [1]. The non-cartilaginous component is typically an osteosarcoma, fibrosarcoma or malignant fibrous histiocytoma with rare instances of leiomyosarcoma, giant cell tumor and rhabdomyosarcoma [2].

Conclusion: We report our rare instance of squamous dedifferentiation of a dedifferentiated chondrosarcoma along with other five cases that have been reported so far in the literature.
\end{abstract}

() 2020 Humaid Al Farii. Hosting by Science Repository.

\section{Introduction}

Cartilaginous tumor such as chondrosarcomas are well-known tumor for a phenomenon called dedifferentiation in which a benign or low-grade malignant tumor undergoes a transformation into a higher-grade tumor. However, the rate of dedifferentiation varies between studies, but usually range from 7-20\% [3]. Rates are also varying depending on the location of the tumor, central malignancies tending to dedifferentiate more than peripherally located similar tumors [3]. However, the pathogenesis of that rare process is still being investigated nowadays. Two schools of thought are mainly focused on: a direct transformation from a welldifferentiated cartilage lesion or a transformation within dense fibrotic reaction surrounding necrotic areas at the margin of the chondral component [3].

Dedifferentiated chondrosarcoma is usually diagnosed in $10 \%$ of all the cases of chondrosarcomas [3]. This histological type is typically present with a faster growth and a rapid metastatic spread in the 5th or 6th decade in the femur, pelvis or humerus most commonly [4]. Approximately $30 \%$ of patients will present with a pathologic fracture and $20 \%$ will present with metastases at the time of diagnosis [3]. MRI studies tend to be more sensitive for revealing the presence of a dedifferentiated component usually represented by a sharply defined osteolytic area next to a hyperintense chondral component [3]. Three components have also been described in the literature: a biphasic pattern (type 1), multiple areas with matrix mineralization representing an underlying residual enchondroma (type 2) or associated important zone of necrosis (type 3) [3].

Metastases are strongly associated with poorer outcomes in chondrosarcoma [5]. They usually consist of the only high-grade anaplastic component. Most common location includes the lungs in about $70-82 \%$ of the cases, the viscera in $20 \%$ and the skeleton in $10 \%$. Tumor location has been reported as an independent risk factor for metastases and death, particularly the pelvis [6]. No proposed guidelines exist regarding the treatment of those metastases. Our case report and literature review include an interesting squamous dedifferentiation of a dedifferentiated chondrosarcoma in a patient that was recently encountered at our oncologic center.

\section{Case Report}

72 years-old African American man who presented to our local center with a 3-month history of mechanical pain in the right lower thigh that progressively worse, especially after a twisting motion the week prior to presentation. The patient reported a 10 pounds involuntary loss, but no low-grade fever, chills or night sweats. The patient was previously 
diagnosed with dyslipidemia, exertional angina, hypertension and hypothyroidism. He had no allergies and was taking Aspirin, Rosuvastatin, Losartan and Levothyroxine.

Physical examination reveals an atrophy of the right quadriceps with a soft tissue mass palpable anteriorly and deeply seated under the facia, attached to the bone right above the patella. His neurological examination including femoral, sciatic, tibial and peroneal nerves are intact, the distal pulses are palpable. He has no palpable lymphadenopathy and has a full range of motion of both knee and hip. He was observed to have antalgic gait.
A radiograph of the involved area showed a lytic lesion with cortical breakthrough and possible associated calcification and scalloping, most consistent with a cartilaginous malignancy (Figures 1A \& 1B). A Computed Tomography (CT) scan and Magnetic Resonance Imaging (MRI) showed a mix of lytic and blastic lesion with calcification, scalloping and an anteromedial associated soft-tissue mass (Figures 1C1F). A chest-abdomen-pelvis CT scan and Proton Emission Tomography (PET) scan failed to reveal any metastases or another primary neoplasm.

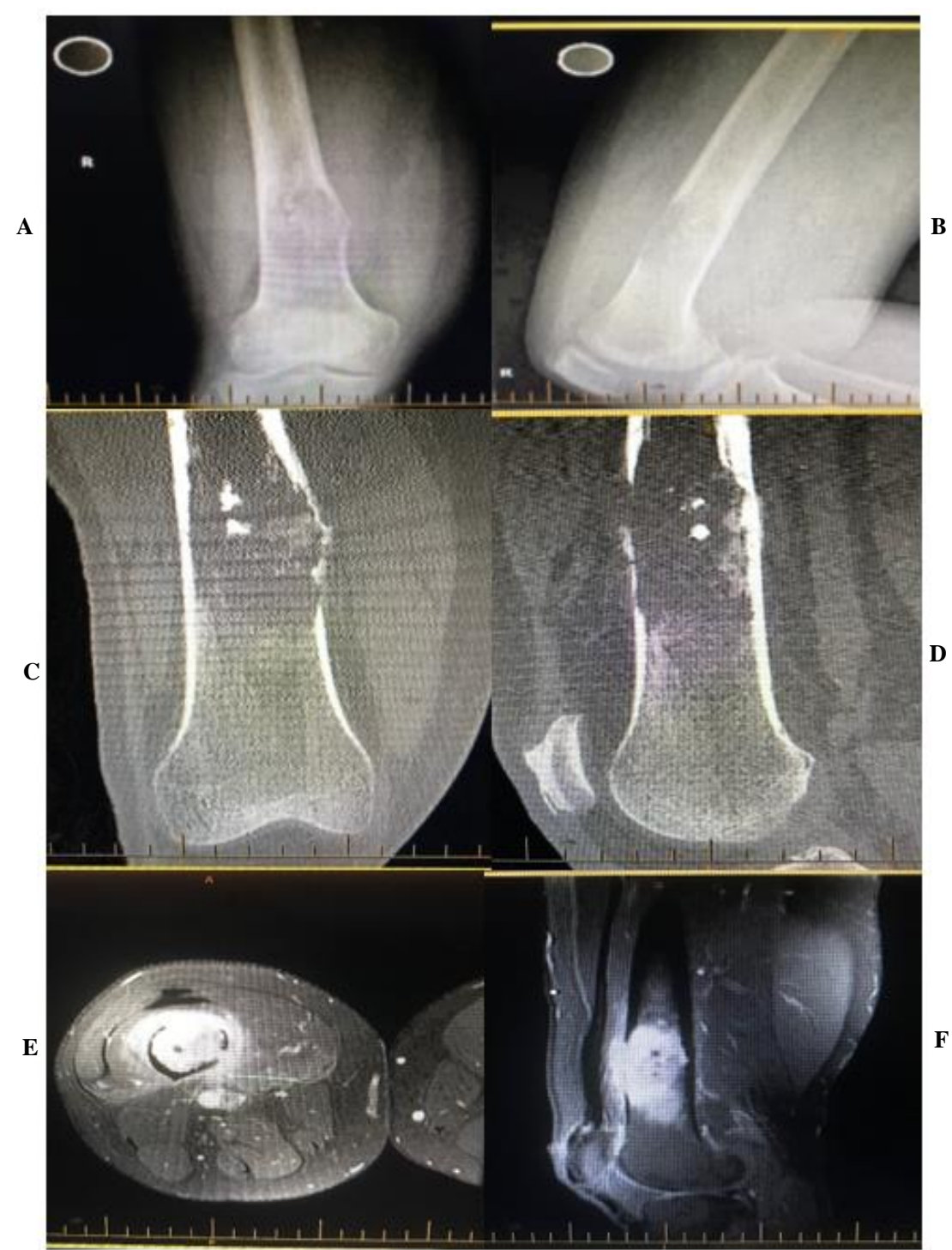

Figure 1: The radiological features of Right distal femur tumor showing aggressive bony lesion within metadiaphysis area with cortical destruction and tumor extension into surrounding muscles. A) \& B) X-ray, C) \& D) CT scan, E) \& F) MRI.

The impression was dedifferentiated chondrosarcoma impending fracture. He then underwent a CT-guided core biopsy that made a preemptive diagnosis of high-grade sarcoma with bone formation through medial vastus medialis muscle compartment. A biopsy result showed high-grade bone forming sarcoma with no cartilaginous component, so the impression of either high grade osteosarcoma or dedifferentiated chondrosarcoma with osteosarcomatous differentiation. A decision was thus made at this point to perform surgery right away. The patient subsequently underwent a right distal femoral and proximal tibia wide resection of the sarcoma and a complex distal femoral reconstruction with a Global Modular Replacement System (GMRS) mega-prosthesis (Stryker Company ${ }^{\circledR}$ ). His pathology results confirmed negative margins 
for dedifferentiated chondrosarcoma with osteosarcomatous and epithelioid (squamous) dedifferentiation (Figure 2). The specimen

positive expression for CK AE1/AE3 in the epithelial component, and negative expression CD31 and ERG.

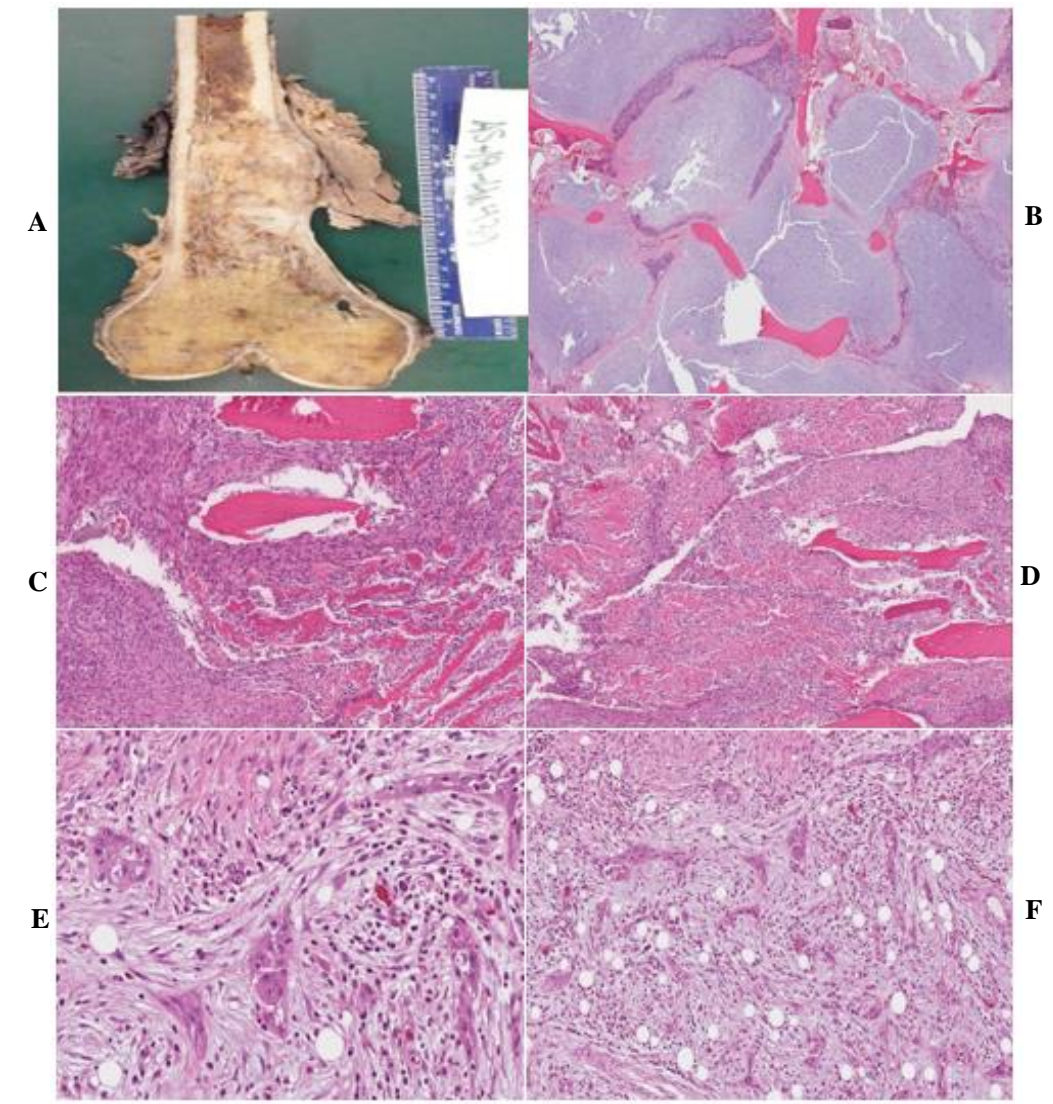

Figure 2: Histological features of tumor: A) Distal femur macroscopic feature of $6.0 \mathrm{~cm}$ (superior-inferior) $* 4.5 \mathrm{~cm}$ (anterior-posterior) $* 3.5 \mathrm{~cm}($ mediallateral) intramedullary mass, and B)-F) microscopic feature of dedifferentiated chondrosarcoma with osteosarcomatous and epithelioid (squamous) dedifferentiation.

His case was reviewed at the tumor board and no recommendation for adjuvant chemotherapy in the presence of overall poor prognosis. The patient was seen in the clinic 6 weeks and 3 months postoperatively with repeat $\mathrm{CT}$ chest that showed negative for lung nodules.

\section{Discussion}

Epithelial tissue dedifferentiation has rarely been reported in the literature. To our current knowledge, only 5 other articles reporting a similar dedifferentiated component to exist. Three also include a squamous transformation, 1 describes a glandular epithelium and the last report an adamantinoma-like basaloid epithelium dedifferentiation.

A first publication in 1985 by Ling and Steiner reported a case of a 68 years old female that presented with a 2-month history of left shoulder pain [7]. The radiological investigation demonstrated a destructive lesion in the proximal end of the humerus, with several patchy radiopaque foci, a partially destroyed cortex and a soft tissue mass extending medially. A bone scan was negative for metastases. A Tikhoff-Linberg operation was performed, including the resection of the upper third of the left humerus, the entire scapula and lateral segment clavicle as well as adjacent soft tissues. Histological and immunological studies showed a dedifferentiated chondrosarcoma with large areas of squamous cell carcinoma. The patient unfortunately died more than 3 years after the symptoms with no evidence of local recurrence or metastases who however refused investigations for mental confusion prior to her death and an autopsy was not performed.

Then Shiraishi et al., report in 2005 describe the story of a 53 years old male who was investigated for a year history of right thigh pain [8]. Radiograph was performed and demonstrated a mix of lytic and sclerotic lesion in the femoral diaphysis with periosteum reaction and change in cortical border in addition to lung nodules that were discovered. A curettage of the femoral lesion was performed and a grade 1 chondrosarcoma. Hemipelvectomy and chemotherapy were started. Final histopathology showed a lesion composed undifferentiated spindle cell sarcoma with small foci of chondrosarcoma and small foci squamous cell carcinoma with prominent keratin pearl formation. Unfortunately, after a few weeks of the surgery, cutaneous metastases appeared and a large mass abutting the right pulmonary artery was found on computed tomography. The patient quickly passed away 6 months after the operation.

In 2014, Zhang et al. describe the case of 74 years old female with a few weeks' history of right thigh pain and inability to walk [9]. Radiographs of the femur showed a radiolucent lesion with stippled calcifications and 
deep scalloping of the endosteum. A complete resection of the femur with negative margins was performed dedifferentiated chondrosarcoma was diagnosed on the final histopathology and he remained disease free for a year. A clinical recurrence confirmed on CT scan and biopsy was discovered during the follow up visits. He was thus started on underwent a lymphadenectomy that revealed a component of squamous cell carcinoma in 2 of 8 superficial femoral lymph nodes and 1 of 2 external iliac lymph nodes. He remained disease free after the procedure and was sent home 4 months after rehabilitation. The review of the initial biopsy with the metastases showed an identical appearance of the dedifferentiated component and of the keratinized squamous cell carcinoma.

Short after, Jour et al. report a 64 years old male presenting with a right femoral uptake on a bone scan for a post-operative follow-up for prostate cancer resection [10]. An MRI showed an enhancing chondroid tumor within the femoral shaft without evidence of soft tissue extension or cortical breakthrough. A biopsy revealed an intermediate grade chondrosarcoma. He later underwent a right femoral resection with allograft reconstruction with no adjuvant treatment. He remained disease free until 9 months post-operatively, when a local recurrence at the distal end of the previous tumor bed was found. A resection of the allograft with a total knee replacement was then performed with no additional treatment. Final histopathology showed a conventional chondrosarcoma juxtaposed to a region in the distal aspect of the tumor composed of numerous anastomosing nests and cords of malignant epithelial cells. He remained free of disease after the second operation.

The last case reported by Gambarotti in 2017 describe a 78 years old male with treatment resistant left knee pain though to be secondary to osteoarthritis [11]. A cartilage-like lesion in the tibia with thickened, scalloped cortex and 2 ill-defined lesions causing cortical breakdown were demonstrated on x-rays. Additional imaging studies revealed a soft tissue extension and left upper lobe nodule. He underwent a resection of the proximal tibia with wide margins. Final diagnosis of dedifferentiated chondrosarcoma with epithelial("adamantinoma-like") features was made. Unfortunately, multiple metastases with progression of the lung nodule and local recurrence were found briefly after the initial surgery. A second operation was done for the local recurrence. The patient finally passed away 8 months from the initial resection.

\section{Conclusion}

In conclusion, although dedifferentiated chondrosarcoma with epithelial component differentiation is still a rare pathological entity, the Orthopedic surgeons, Oncologists and pathologists should be aware about its existence.

\section{Funding}

None.

\section{Conflicts of Interest}

None.

\section{Discloser}

We had full access to all the data in the study and take responsibility for the integrity and accuracy of the data, as well as the decision to submit for publication.

\section{REFERENCES}

1. Dahlin DC, Beabout JW (1971) Dedifferentiation of low-grade chondrosarcoma. Cancer 28: 461-466. [Crossref]

2. Al Saanna G, Bovee J, Hornick J, Lazar A (2018) A review of the WHO classification of tumors of soft tissue and bone.

3. Mavrogenis AF, Papagelopoulos PJ (2018) Bone: Dedifferentiated Chondrosarcoma.

4. Wittig JC (2018) Dedifferentiated Chondrosarcoma.

5. Strotman PK, Reif TJ, Kliethermes SA, Sandhu JK, Nystrom LM (2017) Dedifferentiated chondrosarcomas: A survival analysis of 159 cases from the SEER database (2001-2011). J Surg Oncol 116: 252257. [Crossref]

6. Gelderblom H, Hogendoorn PC, Dijkstra SD, van Rijswijk CS, Krol $\mathrm{AD}$ et al. (2008) The clinical approach towards chondrosarcoma. The Oncol 13: 320-329. [Crossref]

7. Ling LL, Steiner GC (1986) Primary multipotential malignant neoplasm of bone: chondrosarcoma associated with squamous cell carcinoma. Hum Pathol 17: 317-320. [Crossref]

8. Shiraishi J, Mukai M, Yabe H, Shibata R, Yamada T et al. (2005) Primary bone carcinosarcoma: Chondrosarcoma and squamous cell carcinoma with keratin pearl formation. Path Int 55: 504-509. [Crossref]

9. Zhang Y, Mejia AP, Temple HT, Trent J, Rosenberg AE (2014) Squamous cell carcinoma arising in dedifferentiated chondrosarcoma proved by isocitrate dehydrogenase mutation analysis. Hum Pathol 45: 1541-1545. [Crossref]

10. Jour G, Liu Y, Ricciotti R, Pritchard C, Hoch BL (2015) Glandular differentiation in dedifferentiated chondrosarcoma: molecular evidence of a rare phenomenon. Hum Pathol 46: 1398-1404. [Crossref]

11. Gambarotti M, Righi A, Frisoni T, Donati D, Vanel D et al. (2017) Path-Rese and Pract 213: 698-701. 